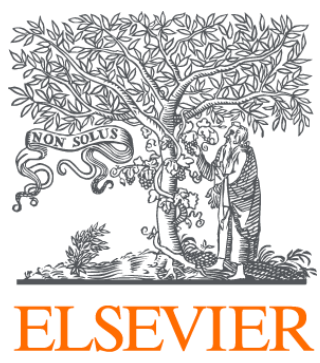

Since January 2020 Elsevier has created a COVID-19 resource centre with free information in English and Mandarin on the novel coronavirus COVID-

19. The COVID-19 resource centre is hosted on Elsevier Connect, the company's public news and information website.

Elsevier hereby grants permission to make all its COVID-19-related research that is available on the COVID-19 resource centre - including this research content - immediately available in PubMed Central and other publicly funded repositories, such as the WHO COVID database with rights for unrestricted research re-use and analyses in any form or by any means with acknowledgement of the original source. These permissions are granted for free by Elsevier for as long as the COVID-19 resource centre remains active. 


\section{Respiratory disease in pregnancy}

\author{
Sophia Stone \\ Catherine Nelson-Piercy
}

\begin{abstract}
Breathlessness in the absence of an underlying pathology is common in pregnancy. Asthma affects about $7 \%$ of women of childbearing age. Treatment is the same as for the non-pregnant population and most drugs are safe in pregnancy. Educating women to continue preventer inhaled corticosteroid therapy will reduce the risk of attacks. Respiratory infections are associated with a higher morbidity in pregnancy and should be treated aggressively. Most chronic pulmonary diseases do not alter fertility. Large reserves in respiratory function allow the fetus and mother to survive without compromise in most cases. The use of chest $X$-rays should not be avoided in pregnancy. Women with a chronic respiratory disease should receive pre-pregnancy counselling and education. Women should be managed in a multidisciplinary setting with the respiratory team. The presence of pulmonary hypertension and cor pulmonale is associated with a high risk of death in pregnancy.
\end{abstract}

Keywords respiratory disease; asthma; pregnancy; pneumonia; tuberculosis

\section{Introduction}

Severe chest disease leading to respiratory failure is uncommon in women of childbearing age but respiratory symptoms are extremely common in pregnancy. This is in part an effect of the physiological adaptation of the respiratory and vascular systems to pregnancy and in part because of limited functional capacity and mobility from diaphragmatic elevation by the gravid uterus in late gestation. Oxygen consumption is increased by $20 \%$ from early pregnancy, minute ventilation by $40-50 \%$ secondary to tidal volume increase, and maternal hyperventilation results in a mild fully compensated respiratory alkalosis (increased arterial $p \mathrm{O}_{2}$ and decreased arterial $p \mathrm{CO}_{2}$, compensatory fall in serum bicarbonate to $18-22 \mathrm{mmol} / \mathrm{l}$; arterial $\mathrm{pH} 7.44)$.

\section{Respiratory symptoms, signs and investigations}

The commonest respiratory symptom in pregnancy is breathlessness. Although not the subject of this article, pulmonary

Sophia Stone mo mRcog is SpR Obstetrics and Gynaecology, St Richard's Hospital, Spitalfield Lane, Chichester, West Sussex PO19 6SE, UK.

Catherine Nelson-Piercy ma FRCP is Consultant Obstetric Physician, Guy's \& St Thomas' Hospitals Trust, London Women's Health Services Directorate, 10th Floor, North Wing, St Thomas' Hospital, Lambeth Palace Road, London SE1 7EH, UK. embolus and cardiac causes are important differential diagnoses, but it is most commonly the increased awareness of the physiological hyperventilation of pregnancy which leads to a subjective feeling of breathlessness. Women most often present in the third trimester but may become symptomatic at any gestation. Classically physiological breathlessness of pregnancy is present at rest or while talking and paradoxically improves with activity. Table 1 summarises other causes. Other presenting symptoms of respiratory disease include chest pain, cough, sputum production, haemoptysis, fever or cyanosis.

On examination, respiratory rate is unchanged by pregnancy. Presence of raised temperature should be noted. Expansion on inspiration reflects tidal volume and is reduced in many respiratory diseases. Percussion note may be dull in the presence of pleural effusion, consolidation, collapse or fibrosis. It is enhanced in the presence of a pneumothorax. Auscultation may reveal wheezes indicative of asthma, fine crepitations in the presence of pulmonary oedema and pleural rubs indicative of inflammatory conditions of the pleura in pneumonia or pulmonary infarction; and bronchial breathing + coarse crackles suggest consolidation. Absent breath sounds occur with pneumothorax or extensive collapse.

A simple non-invasive investigation is transcutaneous oxygen saturation. The normal oxygen saturation is $>95 \%$. A fall in saturation on exercise, e.g. climbing stairs, indicates some form of cardiopulmonary disease and should be investigated further. Measurement of arterial blood gases should be reserved for those who are markedly breathless, those whose oxygen saturations are low at rest or which drop on exercise, and those who appear unwell. Reluctance to perform a chest X-ray in pregnancy may delay a diagnosis. Ionising radiation from a chest X-ray is approximately $0.2 \mathrm{rad}(<5 \%$ of the maximum recommended exposure in pregnancy) and abdominal shielding will reduce fetal exposure further. Sputum should be sent for microbiological examination.

\section{Asthma and atopy}

Asthma is a common chronic inflammatory condition of the lung airways characterised by episodes of reversible bronchoconstriction as a result of various stimuli. It affects up to $7 \%$ of women of childbearing age. It may present with episodes of wheeze, shortness of breath, chest tightness or cough. Signs classically include a diffuse, bilateral, expiratory ( \pm inspiratory) wheeze and tachypnoea but are often absent. There may be an associated personal or family history of atopy and recognised triggers include pollen, dust, animals and infections. Objectively the diagnosis may be confirmed by measuring the peak expiratory flow rate (PEFR) or forced expiratory volume in $1 \mathrm{~s}\left(\mathrm{FEV}_{1}\right)$ as an indication of the degree of bronchoconstriction. A $>20 \%$ diurnal variation on $\geqslant 3$ days in a week for 2 weeks on PEF diary or FEV $\geqslant 15 \%$ increase after a trial of a $\beta_{2}$ agonist or steroid tablets or a similar decrease after 6 minutes of exercise are diagnostic. PEFR and $\mathrm{FEV}_{1}$ are unaffected by pregnancy.

Atopy is often associated with asthma. It has no effect on pregnancy and drugs used in the treatment of hayfever (the antihistamines most commonly used with best safety profile are chlorpheniramine and intranasal beclomethasone) may be safely prescribed. 


\section{Differential diagnoses of breathlessness in pregnancy}

\begin{tabular}{|c|c|}
\hline Diagnosis & Investigations \\
\hline Physiological & Diagnosis by exclusion and typical history \\
\hline Anaemia & Full blood count \\
\hline Asthma & PEFR - response to bronchodilators \\
\hline Pulmonary embolus & $\begin{array}{l}\text { Arterial blood gases }\left(\downarrow p \mathrm{O}_{2} \text { and } \downarrow p\left(\mathrm{O}_{2}\right) /\right. \\
\text { VQ scan CTPA }\end{array}$ \\
\hline $\begin{array}{l}\text { Mitral stenosis, } \\
\text { cardiomyopathy }\end{array}$ & ECG, echocardiogram, chest X-ray \\
\hline Pneumonia & Chest X-ray, sputum culture, serology \\
\hline Pneumothorax & Chest X-ray \\
\hline $\begin{array}{l}\text { Hyperventilation/ } \\
\text { anxiety }\end{array}$ & $\begin{array}{l}\text { Arterial blood gases }\left(\downarrow p \mathrm{CO}_{2} \text { but not }\right. \\
\left.\downarrow p \mathrm{O}_{2}\right)\end{array}$ \\
\hline
\end{tabular}

Table 1

Effect of asthma on pregnancy: Although a number of small studies have suggested an association of asthma with the development of pre-eclampsia, intrauterine growth retardation (IUGR), preterm birth and low birthweights, most pregnancies are unaffected by the effects of asthma. Severe poorly controlled asthma resulting in episodes of maternal hypoxaemia could, however, give rise to such complications. It is therefore of concern that many women stop their medication at the start of the pregnancy because of concerns regarding the safety profile of these drugs for the fetus. In a recent US study, between 5 and 13 weeks' gestation there was a $23 \%$ reduction in use of inhaled corticosteroids, $13 \%$ reduction in short-acting $\beta_{2}$-agonists and a $54 \%$ reduction in rescue steroids.

Effect of pregnancy on asthma: Asthma can worsen, improve or remain unchanged in pregnancy. Endogenous steroids in labour ensure that acute asthma attacks are very uncommon during labour and delivery. There may be a deterioration postpartum.

Management: Pregnancy is an ideal opportunity to optimise therapy. Ideally, this should occur pre-pregnancy. A pre-conceptual visit would allow time to optimise therapy and educate women regarding the importance and safety of continuing their medication to ensure good asthma control throughout pregnancy. Inhaler techniques should be checked and home peak flow monitoring encouraged. Pregnant women should be monitored closely so that any change in course can be matched by an appropriate change in therapy. Smoking should be discouraged.

Drug therapy in pregnancy is essentially the same as for the non-pregnant population and should follow the British Thoracic Society guidelines on the management of asthma. A stepwise approach is recommended (Figure 1). Inhaled $\beta_{2}$-agonists, inhaled and oral steroids, oral and intravenous theophyllines and intravenous magnesium sulphate should be used as normal. Only leukotriene antagonists are not recommended because of limited data, although the guidelines suggest that it is safer to continue these in women who are already taking them and who are resistant to the other therapies.
1. Start treatment at the step most appropriate to initial severity.

2. Achieve early control.

3. Regular review to maintain control by stepping up treatment as necessary; stepping down treatment when control is good.

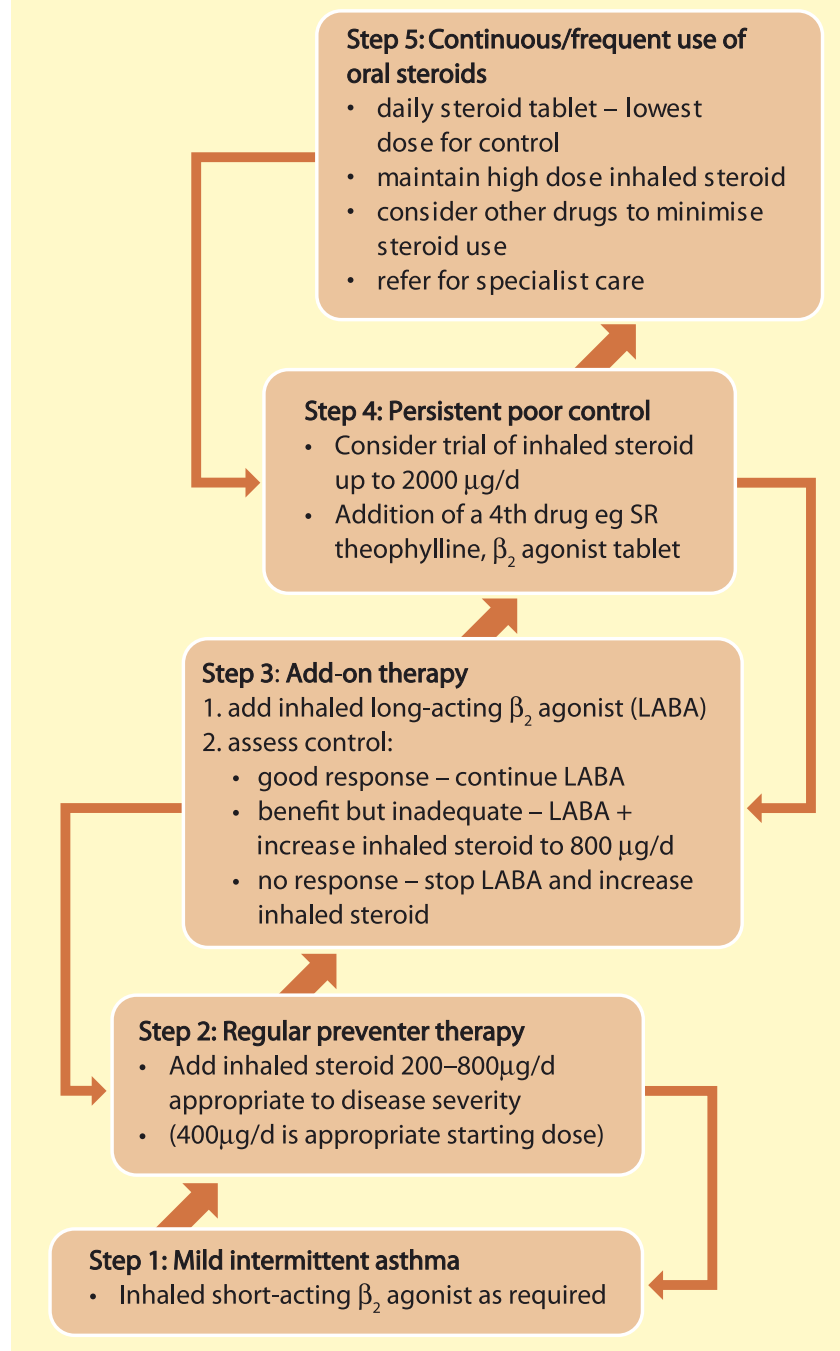

Figure 1 Summary of stepwise management of asthma adapted from the British Thoracic Society Guidelines.

Acute asthma attacks should be managed aggressively as an emergency in hospital. Multidisciplinary care from obstetricians, respiratory physicians and, in severe cases, ITU staff will ensure that appropriate treatment as for the non-pregnant patients is administered (Table 2). Continuous oxygen to maintain saturation above $95 \%$ and continuous fetal monitoring should be instigated. A PEFR of $33-50 \%$ best/predicted indicates acute severe asthma attack; $<33 \%$ is life-threatening. Other concerning signs are $\mathrm{SpO}_{2}<92 \%, \mathrm{O}_{2}<8 \mathrm{kPa}$ with normal $p \mathrm{CO}_{2}$, silent chest, cyanosis, feeble respiratory effort, hypotension, altered consciousness and exhaustion.

If caesarean section is required, regional blockade is preferable to general anaesthesia in women with asthma and opiates should be avoided for pain relief, especially in the presence of an acute 
Treatment of acute asthma (British Thoracic Society Guidelines)

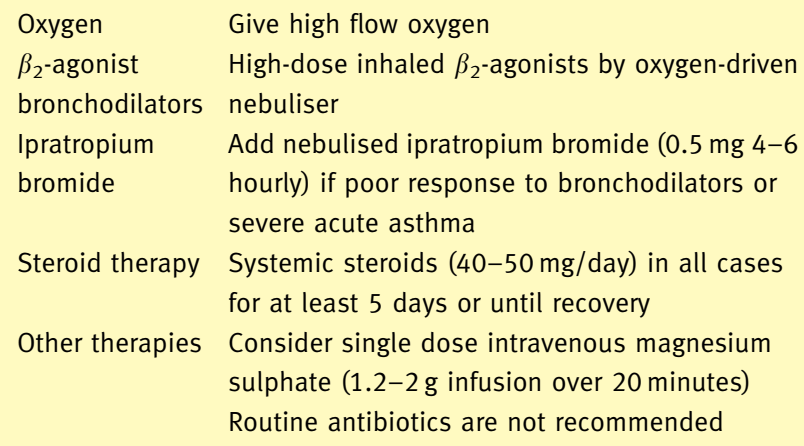

\section{Table 2}

attack. Prostaglandin $E_{2}$ does not cause bronchoconstriction and may used for induction of labour. Prostaglandin $F_{2 \alpha}$ should be used only in extreme circumstances in the management of postpartum haemorrhage and with caution since it may precipitate an acute attack. Likewise aspirin and non-steroidal anti-inflammatory drugs should be avoided, although if aspirin is indicated for pre-eclampsia prophylaxis, and there is a history of aspirin exposure without problems, then it should be safe to use. Encourage women to continue their regular medication in labour. Those women taking $>7.5 \mathrm{mg}$ prednisolone tablets for $>2$ weeks prior to the onset of labour require parenteral hydrocortisone 100 mg 6-8 hourly during labour. Ergometrine has been reported to cause bronchospasm but syntometrine routinely used for active management of the third stage does not.

Postpartum, breastfeeding may reduce the incidence of asthma in the child and should be encouraged. None of the medications for asthma is contraindicated in breastfeeding.

\section{Respiratory tract infections}

Pneumonia occurs in the pregnant population with a frequency equal to that in the general population.

Effects of pregnancy on pneumonia: Pneumonia in pregnancy is often more virulent and mortality is higher than in the nonpregnant population. The spectrum of pathogens is similar to that in the non-pregnant population and the management does not differ. Coexisting maternal disease, including asthma and anaemia, and immunosuppressive therapy increase the risk of contracting pneumonia.

Effect of pneumonia on pregnancy: Severe pneumonia may precipitate preterm delivery and result in low birthweight infants. There is an increased risk of serious maternal complications, including respiratory failure.

Clinical features: Women may present with cough, fever, rigors, breathlessness and pleuritic pain. Signs include fever, purulent sputum, coarse crackles on auscultation and signs of consolidation. Diagnosis may be confirmed with a chest X-ray. Blood and sputum cultures should be taken. Bacterial pneumonia is associated with a raised white blood cell count, although mycoplasma pneumonia is not. Knowledge of the increased mycoplasma activity in the community during an epidemic period may help guide the clinician to the increased likelihood of mycoplasma infection. Mycoplasma and chlamydial pneumonias are diagnosed on serological assays with complement fixation tests.

Management: Supportive measures in the management of pregnant women with pneumonia include oxygen administration if there is evidence of hypoxia, and rehydration especially in the presence of fever. Chest physiotherapy will help clear secretions and aid oxygenation. Oral antibiotic therapy should be commenced for community acquired bacterial infections - oral amoxicillin (0.5-1 g t.d.s.) and clarithromycin (500 mg b.d.) are the current recommended strategies. For severe community acquired or hospital acquired infections intravenous cefuroxime and clarithromycin should be used. Tetracyclines cause discolouration of the teeth in the fetus and should be avoided after 20 weeks' gestation.

\section{Bacterial pneumonia}

Community-acquired pneumonia is most commonly caused by Streptococcus pneumoniae, Haemophilus influenzae and $\mathrm{Myco-}$ plasma pneumoniae. $\beta$-Lactam and macrolide antibiotics are safe in pregnancy and are effective in treating community acquired infections.

\section{Viral pneumonia}

Viral respiratory infections, including varicella, influenza A and severe acute respiratory syndrome (SARS), may present in pregnancy and are associated with a worse outcome than in the non-pregnant population. Current antiviral and respiratory therapies can reduce maternal morbidity and mortality.

The Royal College of Obstetricians and Gynaecologists' guidelines on the treatment of varicella infection in pregnancy recommend that all non-immune pregnant women exposed to varicella should be given zoster immunoglobulin (ZIG) as soon as possible. Those who develop clinical varicella should be treated with aciclovir if within 24 hours of the onset of the rash and after 20 weeks' gestation since mortality and morbidity is higher at advanced gestations. Hospital admission should be arranged in the event of respiratory deterioration, neurological symptoms, haemorrhagic rash and bleeding, or in the presence of a dense rash with mucosal lesions. Delivery during a viraemic period is hazardous with maternal risks of bleeding, thrombocytopenia, disseminated intravascular coagulation and hepatitis. Supportive treatment and intravenous aciclovir may be necessary. There is a high risk of varicella transmission to the newborn with significant morbidity and mortality. Therefore, VZIG should be given to the newborn after delivery (if maternal infection occurred within 5 days pre and 2 days post-delivery). If the neonate develops varicella, aciclovir should be commenced.

Influenza vaccination can reduce the prevalence of hospitalisations among pregnant women during the influenza season and is not contraindicated in pregnancy. 


\section{Pneumocystis pneumonia in association with HIV}

Pneumocystis pneumonia (PCP) is the most common opportunistic infection in patients progressing to acquired immunodeficiency syndrome (AIDS) and continues to carry significant maternal risk to the immunocompromised population. It should be suspected in the presence of profound hypoxia out of proportion to the chest X-ray findings, and bronchoscopy should be considered. Treatment is with high-dose co-trimoxazole (Septrin) \pm pentamidine, usually contraindicated in pregnancy because of the theoretical risks of neonatal kernicterus and haemolysis. However, in the presence of PCP the benefits of treatment outweigh these risks and women with HIV and a past history of PCP or with a CD $4+$ cell count of $<200$ cells $/ \mu$ l should receive prophylactic Septrin or nebulised pentamidine when embarking on pregnancy.

\section{Tuberculosis}

This is an infection caused by Mycobacterium tuberculosis which characteristically causes caseating granulomas, usually involving the lungs as the primary site. The incidence of tuberculosis (TB) worldwide is rising, in part due to the susceptibility to TB of HIVinfected patients, and in the UK is more prevalent amongst Asian and African immigrants. In pregnancy recent studies have suggested a high prevalence (50\%) of extrapulmonary TB (at sites such as lymph nodes, bone, liver, spleen, bone marrow, caecum, nervous system and eye). The patient is often asymptomatic but typically can present with a cough, haemoptysis, weight loss and night sweats. The diagnosis is confirmed with sputum examination for acid-fast bacilli (Ziehl-Neelsen stain). Although pregnancy and TB have little effect on each other, treatment should not be delayed in pregnancy. Because of a rising incidence of drug-resistant $\mathrm{TB}$, treatment is often with prolonged courses of multiple chemotherapeutic agents at the advice of the respiratory physicians. The usual drugs given are rifampicin (no proven adverse fetal effects but risk of maternal hepatotoxicity), isoniazid (in combination with pyridoxine $50 \mathrm{mg}$ /day to reduce the risk of peripheral neuritis), pyrazinamide and/or ethambutol. Liver function should be monitored monthly. Streptomycin is associated with eighth nerve damage and should therefore be avoided. After delivery the neonate should be given prophylactic isoniazid treatment if the mother is sputum positive. The infant should also be vaccinated as soon as possible. Breastfeeding is not contraindicated since very little of the drugs are excreted in breast milk.

\section{Aspiration pneumonia}

This is a complication of gastric regurgitation often at the time of a general anaesthetic in late pregnancy (usually at caesarean section). It may also occur in the event of an excessively high regional block. Increased intra-abdominal pressure, delayed gastric emptying and reduced gastro-oesophageal sphincter tone contribute to the risk. Ranitidine, an $\mathrm{H}_{2}$ antagonist, which reduces gastric acid secretion and an antacid which neutralises the gastric acid are now routinely prescribed for women in labour with risk factors for caesarean section or prior to an elective procedure. The last confidential enquiry into maternal deaths (2000-2002) reported one death from gastric aspiration in an obese woman (BMI >35) following failure to intubate the trachea after induction for general anaesthesia.

\section{Cystic fibrosis}

Cystic fibrosis (CF) is an autosomal-recessive disorder affecting the body's exocrine glands, including the pancreas, sweat glands and lungs. It results from a gene deletion in $70 \%$ of cases and has a carrier frequency of 1 in 25 caucasians. A resultant defect in the CF transmembrane conductance regulator protein causes impaired movement of water and electrolytes across epithelial surfaces. Formerly, CF patients rarely lived to adulthood, but improvements in treatment have meant more women with CF are now reaching reproductive age and wish to become pregnant.

The signs and symptoms of CF vary, depending on the severity of the disease and the degree of bacterial infection. Some cases are very mild and may not be diagnosed until adulthood. CF is characterised by the production of very thick and sticky mucus. The ducts leading from the pancreas become obstructed, causing pancreatic insufficiency, diabetes and malnutrition. About $90 \%$ of CF cases involve the lungs with recurrent or persistent infections, development of bronchiectasis and respiratory failure. A productive cough is almost always present, and very often patients appear barrel-chested. Frequent hospitalisations are a result of recurring respiratory, gastrointestinal and nutritional problems.

Effect of cystic fibrosis on pregnancy: Female fertility may be impaired because of malnutrition or thickened cervical mucus but is usually normal. Men are usually sterile. Most reported series of pregnancies in women with $\mathrm{CF}$ have shown that with careful planning and close monitoring by a dedicated CF team, pregnancy outcomes are favourable. Commonly reported adverse events are IUGR and prematurity, which includes iatrogenic early delivery in unwell women.

Effect of pregnancy on cystic fibrosis: Pregnancy does not affect disease severity or cause deterioration in the patient's condition. Maternal mortality is not significantly greater than non-pregnant age-matched women with CF except in the presence of pulmonary hypertension, cyanosis, arterial hypoxaemia (oxygen saturation $<90 \%)$, severe lung disease $\left(\mathrm{FEV}_{1}<60 \%\right.$ predicted $)$ and/or malnutrition when both maternal and fetal outcome may be poor.

Management: The risk of a child being born with CF is $2-2.5 \%$ if the carrier status of the father is unknown (based on the UK carrier status of 1 in 25) and 50\% if the father is heterozygous for the gene. Therefore pre-conceptual genetic counselling is important. During the pregnancy, regular assessment of fetal growth should be planned to detect early signs of fetal growth restriction.

Ideally women with CF planning a pregnancy should be seen and assessed pre-conceptually. Women with mild disease can then be reassured that pregnancy is safe and liaison may be planned ahead between a CF centre and an obstetrician with a special interest in $\mathrm{CF}$.

Screening for diabetes and baseline lung function tests can be performed. Dietary supplementation and enzyme supplements can be commenced to optimise the patient's condition prior to conception and continued throughout pregnancy. Women with 
CF are at increased risk of developing gestational diabetes. Chest physiotherapy and prophylactic antibiotics may be implemented prior to conception and continued throughout the pregnancy. Infective chest exacerbations should be treated aggressively with antibiotic therapy.

Conversely, if pre-conceptually the patient is found to have pulmonary hypertension complicating the $\mathrm{CF}$, cor pulmonale or an $\mathrm{FEV}_{1}<30-40 \%$, she may be counselled regarding the associated high risk and be strongly advised against pregnancy. Effective contraception should also be discussed in such cases.

In order to achieve a successful outcome, multidisciplinary management aims to ensure adequate maternal nutrition, control of pulmonary infection and avoidance of prolonged hypoxia. Women may develop symptoms of breathlessness or evidence of hypoxia in late pregnancy. Admission for bed rest and oxygen therapy should be arranged. Delivery should be planned if their clinical condition deteriorates further.

There is no contraindication to vaginal delivery but a prolonged second stage should be avoided because of the susceptibility of these patients to pneumothoraces. General anaesthesia should be avoided. There is no contraindication to breastfeeding but women may need to continue nutritional supplements postpartum.

\section{Bronchiectasis}

Bronchiectasis is a sequela of CF, pneumonias and rarer causes such as Kartagener's syndrome. It is characterised by irreversibly dilated damaged bronchi predisposing to persistently infected mucus and bacterial infections. A cough productive of large amounts of sputum is characteristic. The condition is uncommon in the childbearing years but there is some evidence to suggest an association with IUGR when present in pregnancy. Women should be managed jointly with respiratory physicians and their condition can deteriorate in pregnancy. Close attention to postural drainage and physiotherapy will help. Regular sputum cultures and treatment of chest infections are necessary intermittently or continuously if recurrent infections are frequent. The use of bronchodilators may help. As in CF, the presence of pulmonary hypertension and/or hypoxaemia adversely affects prognosis.

\section{Cystic fibrosis lung transplantation recipients}

There are few data regarding pregnancy outcome in women following lung transplant. Of a series of 10 pregnancies in 10 women who were CF lung transplant recipients, five with a long stable interval of $>3$ years between transplant and the pregnancy had favourable outcomes; the remaining five had preterm deliveries. All 10, however, showed progressive decline in lung function and all died of chronic rejection within 38 months of delivery.

\section{Restrictive and fibrotic diseases}

Restrictive ventilatory defects characterised by a reduction in lung volumes and an increase in the ratio of $\mathrm{FEV}_{1}$ to forced vital capacity (FVC) occur when lung expansion is limited because of alterations in the lung parenchyma or because of abnormalities in the pleura, chest wall or neuromuscular apparatus. The majority of pulmonary diseases do not alter fertility. A large reserve in respiratory function allows fetus and mother to survive without compromise in most cases. FVC of $>11$ or $50 \%$ of predicted have been suggested as a cut-off for successful pregnancies and although more severe cases can negotiate pregnancy, patients with severe restrictive lung disease should be advised to avoid pregnancy or consider a therapeutic termination. Associated polycythaemia causing hyperviscosity may add to the thrombotic risk during a pregnancy. Women with an associated kyphoscoliosis are often delivered prematurely because of deterioration in lung function. Mode of delivery tends to be caesarean section because of abnormal fetal presentation. Each case should be assessed individually.

\section{Sarcoidosis}

Sarcoidosis is characterised by non-caseating epithelioid granulomas that may affect any organ system. The aetiology of the disease remains unknown. The disease most commonly involves granuloma formation in the lungs. Other commonly involved organ systems include the lymph nodes (especially the intrathoracic nodes), skin, eyes, liver, heart, and nervous, musculoskeletal, renal and endocrine systems.

The course of sarcoidosis is variable, ranging from self-limited acute disease to a chronic debilitating disease that may result in death. Spontaneous remissions occur in nearly two-thirds of patients, but $10-30 \%$ of patients have a more chronic or progressive course. Because sarcoidosis can involve any organ system, the clinical presentation is often variable. Many patients are asymptomatic but there may be chest symptoms. Other features include erythema nodosum, anterior uveitis, hypercalcaemia, arthropathy, fever or central nervous system symptoms.

Effect of pregnancy on sarcoidosis: According to reported series, sarcoidosis either does not change in pregnancy or if it changes, improves, possibly because of the increased circulating cortisol. However, there is a tendency to relapse in the puerperium. This should not be a contraindication to pregnancy except in severely affected cases pre-pregnancy. Factors indicating a poor prognosis include parenchymal lesions on chest X-ray, advanced radiographic staging, advanced maternal age, low inflammatory activity, requirement for drugs other than steroids and presence of extrapulmonary sarcoidosis.

Effect of sarcoidosis on pregnancy: Sarcoidosis does not adversely affect pregnancy and is not transmitted to the fetus. There is one report of sarcoid granulomata found in the placenta of one patient.

Management: Ideally patients require evaluation before pregnancy to establish chronicity, baseline pulmonary function, inflammatory activity, staging and response to treatment. Systemic steroids should be continued in pregnancy. Angiotensin converting enzyme levels, used as a marker of disease activity, are unreliable in pregnancy. Women should be advised to avoid vitamin $\mathrm{D}$ because of the risk of hypercalcaemia. Intravenous hydrocortisone should be administered in labour in women taking $>7.5 \mathrm{mg} /$ day of prednisolone. 


\section{Wegener's granulomatosis}

This is a rare form of systemic vasculitis in which necrotising granulomatous lesions affect the upper respiratory tract, lungs and kidneys. Without treatment the condition has a poor prognosis. Remission may be achieved with prednisolone and cyclophosphamide, a teratogenic drug. Women should be advised to avoid pregnancy until remission has been achieved and to wait at least 3 months following cessation of cyclophosphamide before conceiving. Cyclophosphamide may also cause ovarian failure.

Pregnancy has been associated with exacerbation of disease activity and cyclophosphamide has been used in the latter half of pregnancy, causing a fetal leucopenia. Antineutrophil cytoplasmic antibody (ANCA) titres are markers of disease activity but their reliability in pregnancy has not been evaluated.

\section{Amniotic fluid embolus}

Amniotic fluid embolism is fortunately rare (1 in 80000) but should be suspected when a woman presents with a sudden onset of hypoxia (with dysnoea, cyanosis or respiratory arrest), collapse, an acute hypotensive crises or disseminated intravascular coagulation or haemorrhage in the absence of any other explanation. Onset is usually during labour, caesarean section or within 30 minutes of delivery. It has also been described during dilatation and curettage procedures. Management is supportive with early involvement of the intensive care team. The aim is to achieve adequate oxygenation with ventilatory support, invasive monitoring to maintain good cardiac output and correction of a coagulopathy. Mortality rate is $>80 \%$. The pathogenesis is not entirely clear. It is thought to be due to the passage of amniotic fluid and debris into the maternal circulation, precipitating an anaphylactic-like reaction. Fetal squames are however present in the circulation of healthy pregnant women without any consequences.

\section{Lung cancer and smoking in pregnancy}

Bronchial carcinoma accounts for $95 \%$ of all primary tumours of the lungs. Although the incidence continues to rise in women, few cases have been reported in pregnancy. The commonest presenting symptoms are cough, chest discomfort and haemoptysis and chest X-ray may demonstrate round shadows. Metastasis has been reported in the pericardium and placenta. Lymphomas may also present with respiratory symptoms.

The strength of association between cigarette smoking and bronchial carcinoma overshadows any other aetiological risk factors and $20-30 \%$ of pregnant women report smoking in the developed world. Few will stop on becoming pregnant but focused counselling by specialist staff has been shown to be more effective than other measures, with more than doubling of cessation rates. The effectiveness and safety of nicotine replacement therapy in pregnancy have yet to be established. Besides maternal morbidity, smoking adversely affects the pregnancy by doubling the risk of premature delivery, both spontaneous and iatrogenic, as a result of placental abruption and lowers the mean birth weight. Reduced ovarian and tubal function and implantation predispose to subfertility, miscarriage and ectopic pregnancy.

\section{Conclusion}

Breathlessness in the absence of an underlying pathology is common in pregnancy, especially in the second and third trimesters. The use of chest X-rays when underlying disease is suspected should not be avoided in pregnancy and most drugs can be used safely in pregnancy. It is important that women with a chronic respiratory disease should receive pre-pregnancy counselling and education regarding the risks of pregnancy and the importance of continuing their medications. It also provides the opportunity to optimise their condition and so reduce adverse pregnancy outcomes. Women should be managed in a multidisciplinary setting with regular review by chest physicians and access to chest physiotherapy if necessary. Liaison with obstetric anaesthetists in the antenatal period will optimise care at delivery with regard to pain relief and reduce anaesthetic risks. Respiratory diseases complicated by pulmonary hypertension and cor pulmonale have a poor prognosis in pregnancy.

\section{FURTHER READING}

British Thoracic Society: Scottish Intercollegiate Guidelines Network. British guideline on the management of asthma. Thorax 2003; 58(suppl):S1-S94.

British Thoracic Society: Guidelines for the management of community acquired pneumonia. Thorax 2001; 56 (suppl IV); 2004; 59: 364-6 (update).

De Swiet M. Respiratory disease. In: de Swiet M (ed): Medical disorders in obstetric practice. 4th ed. London: Blackwell Science, 2002. p. 1-28.

Enriquez R, Wu P, Griffin MR et al. Cessation of asthma medication in early pregnancy. Am J Obstet Gynecol 2006; 1: 149-53.

Gyy KM, Hodson ME, Yacoub MY. Pregnancy in cystic fibrosis lung transplant recipients: Case series and review. J Cyst Fibrosis 2006; 5: $171-5$.

King TE. Restrictive lung disease in pregnancy. Clin Chest Med 1992; 13: 607-22.

Kothari A, Mahadevan N, Girling J. Tuberculosis and pregnancy-Results of a study in a high prevalence area in London. Eur J Obstet Gynecol Reprod Biol 2006; 126: 48-55.

Murphy VE, Clifton VL, Gibson PG. Asthma exacerbations during pregnancy: incidence and association with adverse pregnancy outcomes. Thorax 2006; 61: 169-76.

Nelson-Piercy C. Respiratory disease. In: Nelson-Piercy C (ed): Handbook of obstetric medicine. 3rd edn. London: Informa Healthcare, 2006. p. 65-90.

\section{Practice points}

Asthma

- Counsel women with asthma to continue their medication in pregnancy

- PEFR and FEV1 are unaffected by pregnancy

- Inhaled and nebulised $\beta_{2}$-agonists, inhaled and oral steroids, oral and intravenous theophyllines, and intravenous magnesium sulphate are safe in pregnancy and may be used as in the non-pregnant patient 


\section{Respiratory tract infections}

- Pneumonia in pregnancy is often more virulent and therefore should be treated aggressively

- All non-immune pregnant women exposed to varicella should be given zoster immunoglobulin (ZIG) as soon as possible

- Clinical varicella should be treated with aciclovir if within 24 hours of the onset of the rash and after 20 weeks' gestation

- Risk of TB should be assessed at booking, especially in immigrant women

\section{Cystic fibrosis}

- Joint care with a CF centre is required

- Specialist dietary advice and nutritional supplements should be given, ideally pre-pregnancy

- Infective exacerbations should be treated aggressively

- Prolonged pushing in the second stage of labour should be avoided 